\title{
Sleep duration and blood pressure in Spanish children with obesity
}

\author{
Gloria Pérez Gimeno ${ }^{1}$, Azahara Iris Rupérez ${ }^{1}$, Rocío Vázquez-Cobela ${ }^{2}$, Rosaura Leis ${ }^{3,4}$, \\ Mercedes Gil-Campos ${ }^{5,4}$, María Concepción Aguilera, ${ }^{6,4}$, Ángel Gil ${ }^{6,4}$, Luis Alberto Moreno ${ }^{1,4}$ \\ and Gloria Bueno ${ }^{7,4}$ \\ ${ }^{1}$ Growth, Exercise, NUtrition and Development (GENUD) Research Group, Universidad de Zaragoza, Health \\ Research Institute of Aragón (ISS Aragón), Food and Agriculture Institute of Aragón (IA2), Zaragoza, Spain, \\ ${ }^{2}$ Galicia Nutrition, Growth and Human Development Research Unit, Pediatric Department; Instituto de Investigación \\ Santiago de Compostela (IDIS); Universidad Santiago de Compostela, Santiago de Compostela, Spain, \\ ${ }^{3}$ Galicia Nutrition, Growth and Human Development Research Unit, Pediatric Department; Instituto de Investigación \\ Santiago de Compostela (IDIS); University of Santiago de Compostela, Santiago de Compostela, Spain, \\ ${ }^{4}$ Spanish Biomedical Research Centre in Physiopathology of Obesity and Nutrition (CIBERobn), Instituto de Salud \\ Carlos III (ISCIII), Madrid, Spain, \\ ${ }^{5}$ Paediatric Research and Metabolism Unit, Hospital Universitario Reina Sofia; Instituto Maimónides de Investigación \\ Biomédica (IMBIC), Córdoba, Spain, \\ ${ }^{6}$ Department of Biochemistry and Molecular Biology II, Instituto de Nutrición y Tecnología de los Alimentos, \\ Universidad de Granada; Instituto de Investigación Biosanitaria ibs, Granada, Spain and \\ ${ }^{7}$ Growth, Exercise, NUtrition and Development (GENUD) Research Group, Universidad de Zaragoza, Paediatric \\ Department, Lozano Blesa University Hospital, University of Zaragoza, Health Research Institute of Aragón (ISS \\ Aragón), Food and Agriculture Institute of Aragón (IA2), Food and Agriculture Institute of Aragón (IA2), Zaragoza,
} Spain

\section{Abstract}

Introduction: Hypertension is a public health problem and its prevalence in children is on the rise, often continuing into adulthood and increasing the risk related cardiovascular complications. Sleep plays an essential role in children's growth and several studies suggest that insufficient sleep duration is associated with hypertension in adults. However, few studies have evaluated this association in children. Thus, the aim of the study is to ascertain whether the sleep duration is a risk factor for elevated blood pressure (BP) in children.

Methods: We studied 776 children (5-18 years old), (46.7\% male, 78.8\% 5-13 years, 73.6\% overweight/obesity). Weight and height were measured and body mass index (BMI) was calculated. BP was measured with an electronic manometer to find a $25.3 \%$ of children with an elevated systolic BP (SBP, $>$ P90 ${ }^{\text {th }}$ ) and $14 \%$ with an elevated diastolic BP (DBP, $>$ P90 ${ }^{\text {th }}$ ) (Task Force 2017). Sleep duration was assessed by asking the parents “ ¿How many hours does your child sleep in a typical weekday?”. Weekday sleep hours were considered since they are more representative of children's routines than weekend hours. Hours were converted into a dichotomous variable based on the fulfillment or not of the "Canadian 24-Hour Movement Guidelines for Children and Youth recommendations": 9-11 hours for 5- to 13-year-olds; 8-10 hours for 14- to 17-year-olds.

A spearman correlation analysis was performed between sleep duration, SBP and DBP. Then, a general linear model analysis adjusted for BMI Z-score was done to evaluate differences in SBP and DBP between children following the sleep recommendations. Analyses were stratified by sex and age range (5-13 and 14-17).

Results: In girls 5-13 years, we found a significant negative correlation between sleep duration and body mass index $(\mathrm{BMI})(\mathrm{r}=-0.226$, $\mathrm{p}=0.000)$ and between sleep and systolic BP $(\mathrm{r}=-0.188, \mathrm{p}=0.001)$. Girls $5-13$ years not following sleep recommendations showed significantly higher SBP values than those who followed the recommendations (112 $\pm 11 \mathrm{mmHg} v$ s. $106 \pm 12 \mathrm{mmHg}, \mathrm{p}=0.007)$, independently of BMI. No significant differences were observed in girls 14-17 years nor in males.

Discussion: In the present study, we show that girls 5-13 years with shorter sleep duration show increased SBP values independently of BMI, in contrast to previous studies which showed similar associations that disappeared when adjusting for BMI. Our results support the need for wider strategies in population education so that sleep recommendations are followed by children in the general population.

\section{Conflict of Interest}

There is no conflict of interest 